\title{
La evolución de derecho de aguas en la República Federal Alemana
}

\author{
Marta Oller Rubert \\ Departamento de Derecho Público \\ Universitat Jaume I
}

Sumario: I. LAS COMPETENCIAS SOBRE LAS AGUAS EN LA REPÚBLICA FEDERAL ALEMANA. II. EL DERECHO DE AGUAS ALEMÁN. III. LAS REFORMAS DE LA WASSERHAUSHALTSGESETZ HASTA LA ENTRADA EN VIGOR DE LA DIRECTIVA MARCO DEL AGUA. IV. LA IMPLEMENTACIÓN DE LA DIRECTIVA 2000/60, MARCO DEL AGUA: a) La Directiva Marco del Agua. b) Repercusión en el Derecho alemán estatal. c) Inciso sobre la transposición de la DMA en los Länder.

\section{LAS COMPETENCIAS SOBRE LAS AGUAS EN LA REPÚBLICA FEDERAL ALEMANA}

El Derecho de aguas es en Alemania, como señala BREUER, un campo de tensión (Spannungsfeld) entre las competencias del Bund y las competencias de los Länder ${ }^{1}$. Teniendo en cuenta esta realidad la remisión a la Constitución alemana (Grundgesetz, a partir de ahora GG) es obligada. Así, el artículo 75.1.1.4) GG señala que el Estado (Bund) posee competencia, bajo las condiciones del artículo $72 \mathrm{GG}^{2}$, para dictar Leyes marco (Rahmengesetz ${ }^{3}$ ) en materia de régimen hidráulico (Wasserhaushalt ${ }^{4}$ ), precepto a partir del cual se aprobó la Wasserhaushaltsgesetz, de 27 de julio de $1957^{5}$.

\footnotetext{
${ }^{1}$ Vid. Öffentliches und privates Wasserrecht. Verlag C.H. Beck. München. 2004. Pág. 1. Incluso no debe olvidarse, como así lo señala Hentschel, que ha sido un ámbito clásico de fricción (Reibungspunkt) entre el Derecho administrativo ambiental alemán y la legislación europea, vid. Die europäische Wasserrahmenrichtlinie. Nationale Umsetzungsverpflichtungsplänen und Maßnahmenprogrammen. Dr.Kova. 2005. Pág. 1.

${ }^{2}$ Este artículo regula las llamadas "competencias concurrentes" de la Federación. Ello significa que en el ámbito de la legislación concurrente los Länder tienen competencia para legislar en cuanto la Federación no haya hecho uso de su competencia legislativa a través de una Ley, vid. Wasserhaushaltsgesetz. Kommentar. Kohlhammer. Stuttgart. 2003. Pág. 49.

${ }^{3}$ Esto significa que el Bund no es el único competente para regular dicha materia, sino que esta regulación marco debe completarse con las Leyes de los Länder, vid. KunIG, P «Art. 75, Nota al margen 8» en Grundgesetz. Kommentar. Band.3. München. 1996.

${ }^{4}$ Expresión que es equiparable a la de Wasserwirtschaft, vid. BREUER, R. «Umweltschutz» en Besonderes Verwaltungsrecht. E. Schmidt-Assmann (Hrsg). De Gruyter Recht. Berlin. 2005 Pág. 641; KotULLA. Wasserhaushaltsgesetz...Op. cit. Pág. 35.

El Bundesverfassunsgericht (BVerfG) en su Sentencia de 30 de octubre de 1962 (Tomo 15, Págs.15) definió este concepto como «el conjunto de normas jurídicas para la administración y ordenación de las aguas que discurren en el medio natural». También en este sentido se pronunció el Bundesverwaltungsgericht (BVerwG) en DVBl 2002, Pág. 1474.
}

${ }^{5}$ BGBl I. Págs.110 y ss. Esta ley ha sido de nuevo publicada, introduciendo las oportunas reformas, con fecha de 19 de agosto de 2002, BGBl I. Págs. 3245 y ss. 
Sin embargo antes de entrar en el contenido de la Ley es necesario referirnos brevemente a la evolución de esta inicial competencia de la Federación para aprobar leyes marco, pues ésta se vio limitada - tras la modificación de la Constitución de 27 de octubre de $1994^{6}$ y la introducción de un nuevo apartado $2^{\circ}$ al artículo 75 - reduciéndose significativamente la amplitud de la Federación para dictar normativa-marco a favor de los Länder ${ }^{7}$. También como resultado de esta reforma se introdujo, con gran trascendencia desde el punto de vista formal, la protección del medio ambiente en el artículo 20.a) GG, configurada aquélla como un fin del estado $\left(\right.$ Staatsziel $\left.^{8}\right)$. Este precepto, al igual que ocurre en nuestro Derecho, se configura como el paraguas bajo el cual se desarrolla el Derecho de aguas como conjunto de normas que tiene por objeto el recurso natural de las aguas, como parte del medio ambiente ${ }^{9}$.

Así una primera reflexión acerca de la distribución de competencias en materia de aguas sitúan de manera concurrente las competencias legislativas de la Federación y los Estados federados que se distribuyen de la siguiente forma: en un primer nivel se sitúa el Bund que dicta el marco legislativo, y en un segundo nivel, de desarrollo y ejecución, están los Länder ${ }^{10}$.

Además, en el Derecho de aguas alemán deben diferenciarse diferentes tipos de competencias según se trate un ámbito determinado. Por ello en el caso de que se trate de vías navegables (Wasserstrassen) el Estado posee una competencia concurrente, según el art.74.1.21) GG, en base a la cual se aprobó la Bundeswasserstrassengesetz (WaStrG), de 2 de abril de $1968^{11}$, competencia que también le permitió regular la navegación fluvial interna a través de la Binnenschiffahrtsaufgabengesetz (BinSchAufgG) ${ }^{12}$.

Por otra parte, también los municipios (Gemeinde) y las mancomunidades (Gemeindeverbände) poseen en el Derecho de aguas alemán importantes competencias. Ello deriva del reconocimiento a la autonomía local y de

\footnotetext{
${ }^{6}$ BGB1.I. Pág. 3146

${ }^{7}$ BGB1 I, Pág. 3146. La reforma supuso la introducción de un nuevo apartado al art.75, de forma que según el nuevo art.75.2 GG sólo excepcionalmente las disposiciones-marco (Rahmenvorschriften) podrán entrar en detalle o contener regulaciones de aplicación inmediata, sin definir — como se criticaqué se entiende por casos excepcionales.

${ }^{8}$ Vid. Wolf, J. Umweltrecht. Verlag C.H.Beck. München. 2002. Págs. 125 y ss.

9 Al respecto el comentario jurisprudencial de la BVerwG de 19 de diciembre de 1997, en $Z f W$ 1998.Pág. 495. Entre nosotros el profesor EMBID IRUJO establece esta indudable relación entre la protección del medio ambiente prevista en el art.45 CE y la protección de las aguas, vid. El nuevo derecho de aguas: Las obras hidráulicas y su financiación. Civitas Madrid. 1998. Págs. 74 y 75.
}

${ }^{10}$ Vid. Berendes. Das Abwasserabgabengesetz. Beck. München.1995. Pág. 232.

11 BGB1 II. Pág. 173. La versión vigente es la publicada el 4 de noviembre de 1998 (BGB1 I. Pág. 3294), que fue modificada por la Ley de 18 de junio de 2002 (BGB1 I. Pág. 1914). La última modificación es de 3 de mayo de 2005 (BGBl I. Pág. 1224).

${ }^{12}$ En su redacción de 5 de julio de 2001 (BGB1 I. Pág. 2026). 
la garantía de las mismas que el art.28.2) $\mathrm{GG}^{13}$ y las Landesverfassungen ofrecen. A partir de ello los municipios son competentes para regular bajo su propia responsabilidad todos los asuntos de su interés, pues los entes locales y su ordenamiento jurídico local obliga y vincula también, particularmente, en el ámbito de lo que se denomina «öffentliche Daseinsvorsorge», concepto dentro del cual se sitúan servicios como la eliminación de aguas y regulaciones como la de las aguas residuales y la conexión al alcantarillado, así como las ordenanzas sobre aguas potables ${ }^{14}$.

\section{EL DERECHO DE AGUAS ALEMÁN}

El Derecho de aguas abarca en la República Federal Alemana el Derecho de la economía del agua o de régimen hidráulico (Wasserwirtschaft), o, en otras palabras, podemos desglosar esta materia en el Derecho de régimen hidráulico (Wasserhaushalt) y en el Derecho de las vías fluviales (Wasserwegerecht $)^{15}$.

El Derecho de aguas está formado en su núcleo por la Wasserhaushaltsgesetz, de 27 de julio de 1957 (WHG) y las Landeswassergesetze $e^{16}$. El objetivo inmediato de las normas anteriores es regular los usos de las aguas y por tanto también la ordenación de las autorizaciones y concesiones sobre los mismos.

Los orígenes ${ }^{17}$ de la Wasserhaushaltsgesetz se remontan, recientemente, al año 1949 cuando comienzan a incrementarse las necesidades de agua y con

\footnotetext{
${ }^{13}$ Vid. SchосH, F., «En relación a la situación de la autonomía local tras la «Sentencia-Rastede» del Tribunal Constitucional Federal» en Documentación Administrativa. Núm.234. 1993. Págs.281 a 321.

${ }^{14}$ Vid. Schmidt-Assmann en $N V w Z$. 1987. Pág. 265.

${ }^{15}$ En este sentido Kotulla. Wasserhaushalts... Op. cit. Pág. 35.

${ }^{16}$ En este sentido Breuer, R. Öffentliches und privates... Op. cit. Pág. 7.Las Leyes de aguas de los Länder son las siguientes: Wassergesetz Baden-Württemberg (BWWG), en su redacción de 1 de enero de 1999 (GB1.Pág. 1), Wassergesetz Bayern (BayWG) en su redacción de 24 de abril de 2001 (GVB1. Pág. 140), Wassergesetz Berlin (BlnWG), en su publicación de 3 de marzo de 1989 (GVBl. Pág. 605), Wassergesetz Branderbug (BbgWG) de 13 de julio de 1994 (GVBl Pág. 260),Wassergesetz Bremen (BrrWG), en su redacción de 2 de julio 2002 (GB1. Pág. 245), Wassergesetz amburg (HbgWG) de 20 de junio de 1960 (GVB1. Pág. 335), Wassergesetz Hessen (HeWG), en la redacción de 25 de septiembre de 1996 (GVBl I. Pág. 384), Wassergesetz Mecklenburg-Vorpommern (MVWG), de 30 de noviembre de 1992 (GVBl. Pág. 669), Wassergesetz Niedersachen (NdsWG), en su redacción de 25 de marzo de 1998. (GVBl. Pág. 348), Wassergesetz Nordrhein-Westfalen (NWWG), en su redacción de 25 de junio de 1995 (GV. Pág. 926), Wassergesetz Rheinland-Pfalz (RPWG) en su redacción de 14 de diciembre de 1990 (GVBl. Pág. 11), Wassergesetz Saarland (SIWG), en su redacción de 3 de marzo de 1998 (AmtsBl. Pág. 306), Wassergesetz Sachsen (SächsWG), en su redacción de 21 de julio de 1998 (GVBl. Pág. 393), Wassergesetz Sachsen-Anhalt (LSAWG), en su redacción de 21 de abril de 1998 (GVBl. Pág. 186), Wasergesetz Schleswig-Holstein (SHWG), en su redacción de 13 de junio de 2000 (GVBl Pág. 490), Wassergesetz Thüringen (ThüWG), en su redacción de 4 de febrero de 1999 (GVBl. Pág. 114).

${ }^{17}$ Sobre los antecedentes más remotos de la WHG, vid. CZYCHOWsKI/ ReINHARDT. Wasserhaushaltsgesetz. Kommentar. Verlag C.H.Beck. München. 2003. Págs. 43 y 44; SPARwasser/Engel/VoßKuhle. Umweltrecht. C.F. Müller. Heidelberg. 2003. Pág. 512.
} 
ello crecen también los vertidos de aguas residuales aumentando en gran manera la contaminación de los ríos; es por ello que en ese año la Administración para la Economía (precedente del actual Ministerio de Economía) comenzó a preparar una regulación estatal sobre aguas. Sin embargo no sería hasta 1952 cuando el tema de Derecho de las aguas pasó a estar a la orden del día de la política, reconociéndose por entonces la competencia del Gobierno federal para dictar normas marco en este ámbito. El 4 de febrero de 1956 el Gobierno presentó un proyecto de Ley para la ordenación del régimen hidráulico que fue aprobado por el Parlamento el 27 de junio de 1956 y que debía entrar en vigor, de acuerdo con el artículo 45, el 1 de marzo de 1959. Finalmente la Ley entró en vigor en el año 1960 consecuencia de la primera de las reformas, como veremos.

La WHG consta de 45 artículos, estructurados en 6 títulos, y de dos anexos. El primer título (arts.1.a) a 22 WHG) contiene las disposiciones comunes para las aguas, el segundo título (arts. 23 a 32) se dedica a las disposiciones sobre las aguas superficiales, el tercer título se refiere las aguas costeras, el título cuarto contiene las disposiciones para las aguas subterráneas, el quinto se refiere a la planificación hidrológica, al Libro registro de las aguas, y a la comunicación e intercambio de información, finalmente el título sexto contiene las prescripciones sobre multas. El Anexo I recoge, en relación con el artículo 1.b)1.3) WHG, las cuencas hidrográficas en las que organiza el territorio alemán de acuerdo a las exigencias de la Directiva Marco del Agua; mientras que el Anexo II, en relación con el artículo 7.a).5) WHG, contiene los criterios para determinar el denominado «estado de la técnica» ${ }^{18}$.

La WHG se desarrolla a través de diversos reglamentos destinados a ampliar aspectos concretos en ella regulados. Así, el artículo 6.a) WHG sobre aguas subterráneas se desarrolla por la Grundwasserverordnung, de 18 de marzo de $1997^{19}$, en trasposición de la Directiva 80/68/CEE, de 17 de diciembre, sobre la protección de las aguas subterráneas frente a la contaminación a través de determinadas sustancias peligrosas; el artículo 7.a) WHG, relativo a los requisitos para el vertido de aguas residuales, se amplía a través de la Abwasserverordnung, en su nueva publicación de 29 de septiembre de $2001^{20}$ y el artículo 19.a.2.2) WHG, que regula las autorizaciones de las instalaciones destinadas a transportar sustancias peligrosas para las aguas, por la Verordnung über wassergefährdende Stoffe bei der Beförderung von Rohrleitungsanlagen, de 19 de diciembre de $1973^{21}$.

\footnotetext{
${ }^{18}$ Como señala J.Esteve PARdo las expresiones «estado de la técnica», «mejor tecnología disponible», «mejores técnicas conocidas», etc., pueden reconducirse a lo que se ha llamado cláusula técnica, cada vez de mayor importancia en el seno del Derecho Ambiental, pues con ella se muestra uno de los rasgos propios del derecho vigente y es la necesidad constante de adaptación al desarrollo tecnológico, vid. Derecho del Medio Ambiente. Marcial Pons. 2005. Pág. 149.

${ }^{19}$ BGB1 I. Pág. 542.

${ }^{20}$ BGB1 I. Pág. 2440. Última modificación de 2 de julio de 2002. (BGB1 I. Pág. 2497).

${ }^{21}$ BGB1 I. Pág. 1946. Última modificación de 5 de abril de 1976. (BGB1 I. Pág. 915).
} 
Otras normas cuyo contenido tiene un carácter más específico y sectorial son la Wasch- und Reinigungsmittelgesetz (WRMG), en su redacción de 5 de marzo de $1987^{22}$, de acuerdo a la cual sólo pueden ponerse en circulación aquellos detergentes y productos de limpieza que, tras su uso, no produzcan una merma o perjuicio de las aguas, en particular a la vista de la regulación sobre el ciclo natural y de la reglamentación sobre las aguas potables. También hemos de citar la Wassersicherstellungsgesetz (WasSG), de 24 de agosto de $1965^{23}$; se trata ésta de una Ley para la defensa de las aguas y para asegurar su disposición para una serie de casos y situaciones básicas como puede ser el abastecimiento de la población civil, el transporte de las aguas residuales, etc.; por otra parte la Wasservebandgesetz de 12 de febrero de $1991^{24}$, regula las Wasser-Bodenverbände (Confederaciones hidrográficas) como instituciones públicas independientes que bajo su propia responsabilidad realizan servicios de carácter hídrico bajo el control del Estado Federal. Además, de nuevo en base a las competencias para dictar normas marco del Bund que le otorga el artículo 75.1.1.4) GG la Federación aprobó la Gesetz über Abgaben für das Einleiten von Abwasser in Gewässer (Abwasserabgabengesetz-AbwAG) de 13 de septiembre de $1976^{25}$ — que entró en vigor el 1 de enero de 1981 - Esta ley regulaba una figura impositiva, el canon de aguas residuales (Abwasserabgabe) a partir del cual todo aquel que produjera agua contaminada debía pagar una cantidad determinada, en aplicación directa del principio «quien contamina, paga» ${ }^{26}$.

Al inicio de este epígrafe aludíamos también como parte de Derecho de Aguas al Wasserwegrecht. Bajo esta rúbrica se incluyen básicamente dos

\footnotetext{
${ }^{22}$ BGBl I. Pág. 875, última modificación a través de la Ley de 3 de mayo de 2000.(BGBl. I. Pág. 632). Actualmente y debido a las exigencias comunitarias —en concreto al Reglamento 648/2004, de 31 de marzo de 2004, sobre detergentes- existe desde el 12 de mayo de 2005 un proyecto de Ley sobre la tolerancia del medio ambiente de detergentes y productos de limpieza y con fecha de 20 de mayo de 2005 un proyecto de reglamento sobre los costes de las actuaciones administrativas de las autoridades ambientales en aplicación del reglamento 648/2004 de detergentes, que pretende sustituir y adecuar la actualmente vigente. Los proyectos pueden consultarse en: www.bmu.de.gewasserschutz/downloads/ doc/355000.php
}

${ }^{23}$ BGB1 I. Pág. 1225. Última modificación a través de la Ley de 14 de septiembre de 1994 (BGB1 I. Pág. 2325).

${ }^{24}$ BGB1 I. Pág. 405.

${ }^{25}$ BGB1 I. Pág. 2721, que fue de nuevo publicada con fecha de 3 de noviembre de 1994 (BGB1 I. Pág. 3370). La última modificación se realizó por la Ley de 9 de septiembre de 2001 (BGB1 I. Pág. 2331). Esta Ley también se dicta como ley marco, por ello cada uno de los Länder ha aprobado asimismo una Ley sobre el canon de aguas residuales, vid. Kotulla. Wasserhaushalt... Op. cit. Pág. 37. Nota 8.

${ }^{26}$ Esta Ley fue reformada por primera vez con la 1.Änderungsgesetz de 14 de diciembre de 1984 cuando se introdujo un nuevo art.12.a), (BGB1 I. Pág. 1515). Posteriormente la 2.Änderungsgesetz de 19 de diciembre de 1986 (BGBl I. Pág. 2619), así como la 3.Änderungsgesetz de 2 de noviembre de 1990 (BGB1 I. Pág. 2425) y la 4. Änderungsgesetz de 5 de julio de 1994 (BGBl I. Pág. 1453) han realizado reformas de carácter jurídico-material como el establecimiento del precio del canon, o la fijación de los criterios para determinar la nocividad...También a partir de la reforma de 1994 entró en vigor esta Ley para los Länder de la antigua República Democrática de Alemania. 
normas: en primer lugar, la Bundeswasserstrassengesetz ${ }^{27}$ (WaStrG), en su redacción de 4 de noviembre de $1998^{28}$. Esta Ley regula las vías navegables internas del Bund que sirven al tráfico general, así como las vías marítimas y todas aquellas cuestiones jurídicas sobre las vías fluviales, y, en segundo lugar, debemos citar la Gesetz über die Aufgaben des Bundes auf dem Gebiet der Binneschifffahrt, en su redacción de 5 de julio de 2001.

Otras normas ya sin el carácter hídrico que caracterizaba las anteriores pero también de consideración por sus repercusiones en el Derecho de aguas son citadas por CZYCHOWSKI y REINHARDT, éstas son la Kreislaufwirtschaftsund Abfallgesetz (KrW-/AbfG) de 27 de septiembre de 1994 - Ley de residuos-, y la Bundes-Bodenschutzgesetz (BbodSchG) —-Ley de protección del suelo- de 17 de marzo de 1998.

\section{LAS REFORMAS DE LA WASSERHAUSHALTSGESETZ HASTA LA ENTRADA EN VIGOR DE LA DIRECTIVA MARCO DEL AGUA}

La Ley de aguas alemana, Wasserhaushaltsgesetz, se aprobó en 1957 y continua vigente hasta hoy, no sin numerosas, y en ocasiones significativas, reformas.

La primera reforma de la WHG, de 19 de febrero de $1959^{29}$, modificó el plazo inicial de entrada en vigor de la WHG, contenida en el artículo 45, hasta el 1 de marzo de 1960. Se inició así una importante sucesión de reformas que afectaron ya al contendido mismo de la WHG y que encabezó la 2.Änderungsgesetz de 6 de agosto de $1965^{30}$, a través de la cual se introdujo, con los artículos 19.a) a 19.f) WHG, la normativa sobre canalizaciones en tubo para el transporte de materiales peligrosos para las aguas y junto con ello se fijaron las normas de control del artículo 21 y se completaron las disposiciones sobre ilegalidades del artículo 41. Con la 3.Änderungsgesetz, de 15 de agosto de $1967^{31}$, los preceptos cuyo objeto era la protección de las aguas costeras, contenida en los artículos 1.1.1.a), 32.a) y b), se ven disminuidos. Más profunda y trascendente fue la 4.Änderungsgesetz, de 26 de abril de $1976^{32}$. Con esta reforma, y a partir del 1 de octubre de 1976, entró, en principio, únicamente en vigor la reforma del artículo 5. Durante la $6^{\mathrm{a}}$ legislatura del Parla-

\footnotetext{
${ }^{27}$ De 2 de abril de 1968 (BGBl II. Pág. 173).

${ }^{28}$ BGBl I. Pág. 3294, última modificación de 18 de junio de 2002 (BGBl I. Pág. 1914)

${ }^{29}$ BGBl I. Pág. 37.

${ }^{30}$ BGBl I. Pág. 611.Por otra parte KotulLa ofrece fechas diferentes, así señala que la 2.Änderungsgesetz es de 8 de agosto de 1964 (BGBl I. Pág. 611), vid. Wasserhasuhalts... Op. cit. Pág. 43.

${ }^{31}$ BGB1 I. Pág. 909.

${ }^{32}$ BGB1 I. Pág. 1109.
} 
mento el Gobierno preparó el proyecto para la aprobación de una 4.Änderungsgesetz, así como dos proyectos más, uno de los diputados de todos los grupos parlamentarios (Bundestagsfraktionen) y otro del Gobierno. Éstos fueron de nuevo revisados en el $7^{\circ}$ periodo de legislatura del Parlamento y dando origen a una nueva 4.Änderungsgesetz. La mayoría de modificaciones contenidas en la 4.Änderungsgestez incumbían a la ordenación pública de los usos de las aguas: en la reforma del artículo 1.a)WHG se introdujo por una parte una modificación de los instrumentos hidráulicos, y por otra parte se introdujeron nuevos instrumentos de control y dirección; sobre todo éstos últimos en relación a las normas sobre emisiones que afectaron a los requisitos para la realización de vertidos de aguas residuales (art. 7.a) WHG), a los requisitos para la eliminación de aguas residuales (art. 18.a) y art.18.b) WHG), a las instalaciones para el almacenamiento, descarga y traslado de sustancias peligrosas para las aguas (arts. 19.g-19.1 WHG), a la constitución y estatus jurídico de las personas delegadas (Betriebsbeauftragten) encargadas de la protección de las aguas (arts. 21.a-21.g WHG) y a los planes hidrológicos como reglamentaciones de las aguas en relación con las inmisiones (art. 36.b WHG).

Posteriormente, a inicios de la década de los ochenta, la Ley para combatir los delitos ambientales ${ }^{33}$ también afectó a la WHG, pues los preceptos que hasta entonces regulaban las sanciones, en concreto los artículos 38 y 39 WHG, endurecidos con la 4.Änderungsgesetz, fueron derogados. En su lugar pasan a ser aplicables los artículos 324, 329.2 y 4), y el artículo 330 del Código Penal alemán (Straftgesetzbuch-StGB). Cuando el 1 de noviembre de 1994 entró en vigor la segunda Ley contra los delitos ambientales ${ }^{34}$ se modificó de nuevo la parte de la ley dedicada a regular los hechos punibles con el fin de mejorar la lucha contra las conductas lesivas del medio ambiente.

La 5.Änderungsgesetz, de 25 de julio de $1986^{35}$, se caracterizó por ser una continuidad de la anterior, confirmándose el principio de precaución, afectando especialmente al artículo 7.a) WHG sobre requisitos mínimos para el vertido de aguas residuales e introduciendo la obligación de disminuir los vertidos de determinadas sustancias peligrosas. Otra de las reformas de 1986 fue la del artículo 19.1.3) WHG que se refiere a las aguas subterráneas. Se introduce así la posibilidad de crear también en relación con estas aguas cuencas de protección para evitar la contaminación producida por la filtración de las sustancias a través del suelo, de los fertilizantes o de las plantas. Continuando con estas aguas también el artículo 19.g.1) WHG amplió el interés por la protección de las mismas exigiendo la construcción de instalaciones adecuadas, la aplicación de tratamientos y uso de sustancias peligrosas... Instalaciones para el almacenamiento, eliminación de aguas de abono, de abo-

\footnotetext{
${ }^{33}$ Gesetz zur Bekämpfung der Umweltkrimialität, 18.StrÄndG, de 28 de marzo de 1980. BGB1 I. Pág. 373.

${ }^{34}$ De 27 de junio de 1994. BGBl I. Pág. 1440.

${ }^{35}$ BGBl I. Pág. 1165.
} 
no líquido y grava ensilada. Finalmente el artículo 36.b.1) WHG, sobre los planes de explotación, mantuvo su finalidad, pero fue modificado para introducir, entre otros, los planes hidrológicos como modo de protección de las aguas.

La 6.Änderungsgesetz, de 11 de noviembre de $1996^{36}$, determinó nuevas y relevantes modificaciones. Esta reforma iniciada durante la $12^{\mathrm{a}}$ legislatura del Parlamento sin que llegara en ese periodo a concretarse, fue finalmente en la $13^{\mathrm{a}}$ legislatura y tras un duro y largo proceso legislativo, aprobada entrando en vigor el 16 de noviembre de 1996, tras la nueva publicación de la WHG de 12 de noviembre de $1996^{37}$. La causa de esta reforma fue la jurisprudencia del Tribunal de Justicia de la Unión Europea que desde 1991 había condenado a diferentes Estados Miembros por la falta de trasposición de las Directivas comunitarias. Así en diversas ocasiones encontró el TJCE que el Reino de Alemania no había cumplido a través de sus leyes o de sus reglamentos jurídicos, entre ellas en la Sentencia de 28 de febrero de 1991, Caso C-131/88, sobre las aguas subterráneas, en la Sentencia de 17 de octubre de 1991, Caso C-59/89, sobre las aguas potables, en la Sentencia de 7 de noviembre de 1996, Caso C-262/95, sobre los vertidos de sustancias peligrosas en las aguas... ${ }^{38}$. Ante tales condenas el legislador reaccionó con la introducción del artículo 6.a) «Supra- und internationale Anforderungen» WHG. El punto clave de esta Sexta Reforma fue, no obstante, la nueva redacción del artículo 7.a) WHG, en el que se sustituye el concepto de «normas de la técnica generalmente reconocidas» (allgemeine anerkannten Regeln der Technik) por el de «estado de la técnica» (Stand der Technik) ${ }^{39}$, concretándose este último criterio a través del actual Anexo II de la WHG. En el plano de

\footnotetext{
${ }^{36}$ BGB1 I. Pág. 1690.

${ }^{37}$ BGB1 I. Pág. 1695.

${ }^{38}$ Vid. EverLING en Jahrbuch des Umwelt- und Technischrecht. 1992. UTR. Band 17. 1992. Págs. 3 y ss; CZYCHOwsKi en Zeitscrhift für Umweltrecht. 1997. Págs. 71 y ss.
}

\begin{abstract}
${ }^{39}$ Sobre la diferencia de uno y otro criterio P. NISIPEANU señala que el concepto de «allgemein anerkannten Regeln der Technik» no aparecía definido en la WHG y que por tanto la única descripción con la que se contaba era con la que, en 1920, dio el Tribunal del Imperio en materia penal. En todo caso se señala como con este concepto jurídico indeterminado el legislador pretendió introducir un criterio amplio y flexible que posibilitara la adaptación de la WHG a los cambios que se producen en el mundo técnico, vid. Abwasserrecht. Verlag C.H. Beck. 1991. Págs. 35 y 36. En cuanto al concepto de «Stand der Technik»es un concepto que aunque no aparecía tampoco definido en la WHG, sí existía una definición en el art.3 VI 1 de la BImSchG, según el cual el «estado de la técnica es el nivel de desarrollo de los procedimientos, instalaciones o formas de funcionamiento que asegura la aptitud de las medidas para limitar las emisiones...». El actual art.3.6 contiene la definición vigente de «estado de la técnica» con un sentido similar, pero para la determinación del mismo se remite a los parámetros fijados en el Anexo de la BImSchG, en la nueva publicación de 26 de septiembre de 2002. BGB1 I. Pág. 3830, última modificación a través del art.1 de la Ley de 25 de junio de 2005.BGB1 I. Pág. 1865. También sobre estos conceptos., vid. Czychowski/ ReInHARdT. Wasserhaushaltsgesetz... Op. cit. Págs. 360 y ss; Sparwasser/ Engel/ VoßKuhle. Umweltrecht. Op. cit. Págs. 60 y 61. Entre nosotros J. Esteve PARDo se ha ocupado del origen y significado de la mencionada terminología alemana en «El componente técnico del Derecho del medio ambiente. Limitaciones y posibilidades de los entes locales» en Derecho del medio ambiente y Administración Local. J. Esteve Pardo (coord.). Civitas. Madrid. 1996. Págs. 456 y ss.
\end{abstract}


las decisiones particulares la ley de modificación corrigió en el artículo 5.1.2) WHG el principio de proporcionalidad. Pues inicialmente el artículo 5.1.1) WHG establecía que las autorizaciones y concesiones se sitúan bajo reserva de que fuera posible establecerse requisitos adicionales según la naturaleza de las sustancias vertidas. Tras la modificación del mencionado precepto tales requisitos adicionales no pueden ser exigidos cuando el cumplimiento de esas condiciones suponga una inversión que impida el disfrute de la autorización o concesión. Otros preceptos afectados por la $6^{\mathrm{a}}$ Novelle fueron los artículos 18.a), 18.b) y 18.c) de la WHG. Respecto al primero de los artículos se incorpora en cuanto a la eliminación de aguas residuales urbanas (Abwasserbeseitigung) la premisa de que para la satisfacción del bien de la comunidad ésta tarea puede ser también realizada a través de instalaciones descentralizadas, y ello con el fin de trasponer al derecho alemán la Directiva 91/271/ CEE sobre el tratamiento de las aguas residuales urbanas (Art. 18.a.1.2. WHG). Siguiendo con este artículo la otra innovación es que en el caso de que se opte para la ejecución de esta tarea por recurrir a terceros-privados (art.18.a.2.3 WHG) los Länder pueden regular bajo qué condiciones (art. 18.a.2.a) WHG) una institución pública puede trasladar tal obligación total o parcialmente, de manera limitada y revocablemente. Además tras la nueva redacción de 1996 las instalaciones para la depuración y tratamiento de las aguas residuales deben en particular cumplir con los requisitos exigidos en el artículo 7.a) WHG, al tiempo que para la construcción y explotación de una depuradora deben tenerse en cuenta el criterio de las «allgemein anerkannten Regeln der Technik», ello difiere de la exigencia del artículo 7.a) WHG que exige un nivel superior que es el que el «Stand der Technik» supone. El artículo 19.h) WHG fue de nuevo redactado con el fin de adecuar el Derecho de aguas al Derecho de los productos de construcción (Bauproduktenrecht), que la Directiva 89/106, de 21 de diciembre, exige. Los cambios de los artículos 21.b) a 21.f) están orientados a mejorar la situación jurídica de los encargados-delegados de la protección de las aguas e incorporar las normas sobre protección de las inmisiones. También se vio afectada por la 6. Änderungsgesetz la regulación de las obras hidráulicas del artículo 31 WHG, según el cual los cursos de aguas naturales no deben ser alterados. Sin embargo debido a la especial situación geográfica de la República federal alemana y de las frecuentes inundaciones que sufren en este territorio se incluyen revisiones para prevenir estas posibles situaciones de catástrofe.

La transposición de la Directiva 96/61, sobre prevención y control integrado de la contaminación (IPPC, IVU-Richtilinie) se realizó a través de la Gesetz zur Umsetzung der UVP-Änderungsrichtilinie, der IVU-Richtilinie und weitere EG-Rischtilinie zum Umweltschutz, de 27 de julio de $2001^{40}$, integrándose el concepto de «Stand der Technik» en el artículo 1.a) WHG.

\footnotetext{
${ }^{40}$ BGB1 I. Pág. 1950 y ss.
} 
Finalmente para concluir esta repaso a lo largo de la historia de la WHG hemos de mencionar las más recientes reformas surgidas en el año 2005. La primera de ellas a través del artículo 1 de la Gesetz zur Verbesserung des vorbeugenden Hochwasserschutzes de 3 de mayo de $2005^{41}$, afectó los artículos 18.a)WHG y 19.a,e,g) WHG, y ,de manera general, el Título IV de la WHG destinado a la protección frente a las inundaciones (arts. 31.1 y ss), la Gesetz zur Umsetzung von Vorschlägen zu Bürokratieabbau und Deregulierung aus den Regionen, de 21 de junio de $2005^{42}$ modificó en su artículo 3 y el artículo 21.b.3 WHG y la última a través del artículo 2 de la Gesetz zur Einführung einer strategischen Umweltprüfung der Richtlinie 2001/42/ EC, de 25 de junio de $2005^{43}$ modificó el artículo 25.a.2 WHG, el art.31.d. al que se le la añadió un nuevo apartado $4^{\circ}$, el artículo 33.a.2 WHG y el artículo 36.7 WHG.

\section{LA IMPLEMENTACIÓN DE LA DIRECTIVA 2000/60, MARCO DEL AGUA}

\section{a) La Directiva Marco del Agua}

La aprobación de la Directiva 2000/60, de 23 de octubre, marco del agua ${ }^{44}$, por parte de la Unión Europea supone un antes y un después en el Derecho de aguas, pues ésta afecta a todos los ordenamientos jurídicos de los Estados Miembros con el objetivo de homogenizar y unificar regulaciones, como bien puso de manifiesto el Seminario Ministerial sobre la política de aguas de la Comunidad, celebrado en Frankfurt en 1988. Posteriormente, en 1995, el Consejo adoptó unas Conclusiones en las que exigía, entre otras cosas, la elaboración de una nueva Directiva marco que estableciera los principios básicos de una política de aguas sostenible en la Unión Europea e invitaba a la Comisión a que presentara una propuesta, a lo que respondió la Comisión con una Comunicación en 1996 en las que se contenían los principios básicos de una nueva política de aguas. Finalmente el Consejo, el 25 de junio de 1996, el Comité de las Regiones, el 19 de septiembre de 1996, el Comité Económico y Social, el 26 de septiembre de 1996, y el Parlamento Europeo, el 23 de octubre de 1996, solicitaron a la Comisión que presentara una propuesta de Directiva del Consejo que estableciera un marco para una política europea de aguas. La Directiva fue finalmente aprobada el 23 de octubre de 2000 y con ella se abrió paso una nueva etapa en el Derecho de aguas.

\footnotetext{
${ }^{41}$ BGB1 I. Pág. 1221.

${ }^{42}$ BGB1 I. Pág. 1667.

${ }^{43}$ BGB1 I. Pág. 1746.

${ }^{44}$ DOCE, Serie L, 327/1, de 22 de diciembre de 2000. Pág. 1. La trasposición de esta directiva debía realizarse, según el art.24.1 DMA, en los Estados Miembros antes del 22 de diciembre de 2003.
} 
Aparecen así nuevos conceptos para unos y no tan nuevos para otros. Es el caso del concepto de demarcación hidrográfica, con una clara inspiración en el modelo de cuenca hidrográfica española ${ }^{45}$, y cuyo objeto, a la vista de la definición de este concepto, era otorgar una protección global de la calidad de las aguas ${ }^{46}$, la necesidad de un órgano de gestión, la mayor implementación de la coordinación en las acciones públicas — por ejemplo hasta el 22 de diciembre de 2004 los Estados tenían de plazo para realizar un inventario de la situación de las aguas en sus territorios, que debían comunicar a la Unión - la introducción del principio de recuperación de costes, implantando ciertos elementos de mercado, en la línea de liberalización y de mercado único que inspira toda la acción de la Unión Europea, así como la necesidad de una mayor participación ciudadana y la transparencia en la información pública ${ }^{47}$. Todas estas exigencias de mano europea deben ser cumplidas en un plazo máximo, así el 2015 es el umbral fijado por la Directiva Marco para conseguir su primordial objetivo: el buen estado ecológico de las aguas.

En definitiva como señala SALZWEDEL se trata de encontrar un equilibrio entre la protección y el uso de las aguas ${ }^{48}$.

\section{b) Repercusión en el Derecho alemán estatal}

La trasposición en el Derecho alemán de la WHG requiere, debido a su organización federal, su transposición tanto en el plano del Bund como de los Länder $^{49}$. En efecto, la circunstancia de que la competencia estatal sobre las aguas sea una competencia concurrente y además limitada a la aprobación de normas-marco, implica que la Federación sólo puede transponer a través de la WHG aspectos esenciales de la DMA, pero no cada una de las exigencias concretas, tarea y obligación que corresponde a los Länder $^{50}$. Nos referiremos aquí ampliamente sólo al primer caso y dejaremos apuntada la situación respecto a los Länder, pues como señala BREUER con la $7^{\mathrm{a}}$ Reforma de la WHG

\footnotetext{
${ }^{45}$ Así en el derecho español y en base al principio de que la cuenca hidrográfica sigue manteniéndose como unidad de gestión del recurso que se considera indivisible, se procedió a introducir la noción de «demarcación hidrográfica» en el art 16.bis del TRLAg definiéndola como «la zona terrestre y marina compuesta por una o varias cuencas hidrográficas vecinas y las aguas de transición, subterráneas y costeras asociadas a dichas cuencas».

46 Vid. Lasagabaster, I. Derecho Ambiental. Parte especial. IVAP. Oñati. 2004. Pág. 51.

${ }^{47}$ Más ampliamente sobre la aplicación de esta Directiva en nuestro entorno, vid. Aplicación en España de la Directiva Europea Marco de Aguas. AA.VV. Ecoiuris-IEH. Madrid. 2003. in totum.

48 Vid. «Wasserrecht im Wandel-Änderungsbedarf» en Änderungsbedarf im Wasserrecht-zur Umsetzung europarechtlicher Vorgaben-. Nomos Verlg. Baden-Baden. 2002. Pág. 19.

49 Vid. KNOPP, L. «Flugebietsmanagement und Verwaltungskooperation» en Änderungsbedarf im Wasserrecht... Op. cit. Pág. 27.

${ }^{50}$ Vid. el informe de la LAWA sobre la trasposición de la DMA en el Derecho Alemán, www.lawa.de/ pub7kostenlos/wrrl/Handlungskjonzept.pdf.
} 
únicamente se ha dado el primer paso a nivel estatal, pero serán necesarias posteriores trasposiciones por parte de los Estados federados ${ }^{51}$.

La transposición, como veníamos diciendo, se realizó a través de la 7. Gesetze zur Änderung des Wasserhaushaltsgesetzes ${ }^{52}$, introduciendo entre otros elementos en el Derecho alemán de aguas el concepto clave en el medio ambiente de desarrollo sostenible ${ }^{53}$ en preceptos como el artículo 1.a) WHG, 1.b) WHG y en el artículo 6.1) WHG en el que se observa el principio de inmisión, según el cual una autorización o cesión no debe concederse cuando ello suponga el menoscabo del bien común. Además y como novedad más relevante se prevé la introducción en el Derecho alemán de un régimen de administración hidrológica, en cuyo ámbito se acentúa particularmente el peso importante de los intereses ecológicos ${ }^{54}$.

En primer lugar se da una nueva redacción al artículo 1.1.2) WHG, en el que se incluyen la definición de las aguas subterráneas y de otros conceptos como el de cuenca hidrográfica, demarcación hidrográfica... Además de enumerase cada una de las demarcaciones en las que se constituye el territorio alemán (art.1.b WHG, en relación con el Anexo 1). Para ello se obliga a los Länder a regular la planificación hidrológica en coordinación entre las cuencas hidrográficas y las demarcaciones a nivel tanto nacional como internacional (art.1.b).2) WHG), trasponiendo así la exigencia de «coordinación de disposiciones administrativas en las demarcaciones hidrográficas» del artículo 3 WHG.

La DMA ha supuesto para la República Federal Alemana el retorno de las «demarcaciones hidrográficas» (Flussgebieteinheit ${ }^{55}$ ) en las que deben aplicarse medidas de coordinación entre los cursos de aguas situados en cuencas hidrográficas (Einzugsgebiet, art.2.13 DMA) de cada Land y también en el caso de cuencas internacionales — caso de la cuenca del Rhein-, todo ello

\footnotetext{
${ }^{51}$ Vid. Öffentliches und... Op. cit. Pág. 15.

52 BGB1 I. 2002.Pág. 1914.

${ }^{53}$ Vid. Beauchamp, G. «Einfürhung in die Tagung-Wasserrecht im Zeichen der Nachaltigkeit» en $\ddot{A} n$ derungsbedarf im Wasserrecht...Op.cit. Pág. 13. Sobre desarrollo sostenible, vid. Das konzept der zukunfstfähigkeiten Entwicklung im Recht. Tübingen. 20002. Págs. 15 y ss. También DREwES, J.E/WEIGERT, B. «Sustainable Development.das neues Denken in der Wirtschaf, Wasser-Abwasser.1998, 699 (701/702), Encuesta de la Comisón «Konzept Nachaltigkeit, Fundamente für eien Gesellschaft von morgen. Zwischenbericht der Enquete-Komission «Schutz des Menschen un der umwelt» des 13.Bundestages. Asunto 1/97 Deutscher Bundestag. Refreat Öffentlichkeitsarbeit. Bonn. 1997.
}

${ }^{54}$ Vid. Kluge, T. «Ansätze zur sozial-ökologischen Regulation der Ressource Wasser- neue Anforederungen durch die EU-Wasserrichtilinie und Privatisierungstendenzen». NetWORKS (Hrsg). Berlin. 2005. Pág. 15. En el mismos sentido K. BERENDES «Die neue Wasserrechtsdordnung» en $Z f W$. 2002.Pág. 197, 211 y ss.

${ }^{55}$ Definidas en el art.2.15 DMA: «la zona marina y terrestre compuesta por una o varias cuencas hidrográficas vecinas y las aguas subterráneas y costeras asociadas, designada con arreglo al apartado $1^{\circ}$ del art.3 como principal unidad a efectos de la gestión de las cuencas hidrográficas». En el art.1.b.1.WHG aparecen enumeradas cada una de las demarcaciones de la República Federal Alemana. 
bajo la denominación de lo que en la República Federal Alemana se ha denominado «Flussgebietmanagement» (art.3 DMA), que se ha traspuesto con la nueva redacción del artículo 1.b) WHG. La gestión de las cuencas, con el fin de lograr los objetivos de la protección de las aguas, se realizará siguiendo pues un plan hidrológico de cuenca (Bewirtschaftungsplan, art.13.1) DMA), que se aplicará a cada cuenca hidrográfica, cuyas determinaciones se especifican en el Anexo II DMA. A través del artículo 36 WHG se ha traspuesto las normas para esta planificación. Un concepto éste que en todo caso no era nuevo tampoco en el ámbito germano, pues aunque con diferentes finalidades también se encontraba de alguna manera recogido en la WHG. La principal diferencia radicaba en que la planificación de la DMA exige una vinculación jurídica obligatoria, mientras que la planificación que la WHG contemplaba solamente un plan técnico, por tanto sin fuerza jurídica ${ }^{56}$; ello obligó a la reforma de diversos artículos entre ellos el artículo 36 WHG, sobre planes hidráulicos marco (Wasserwirtschaftliche Rahmenpläne), el artículo 27 WHG, sobre el régimen de limpieza de las aguas (Reinhalteordnungen) y el artículo 18.1.3) WHG, sobre los planes para la eliminación de agua residuales (Abwasserbeseitungspläne). Por otra parte, el conjunto de actuaciones que debe realizar el Estado Miembro cuando en virtud de actividades de seguimiento $\mathrm{u}$ otros datos se indique que probablemente no se lograrán los objetivos establecidos en el artículo 4 para una masa de agua, se trasponen con el apartado 4 del artículo 36 WHG.

El artículo 4 DMA tiene una especial trascendencia. Este precepto contiene importantes directrices en cuanto a la actuación de las autoridades hídricas - pues el deterioro y empeoramiento de las aguas va a limitar en gran medida el uso de los recursos hídricos- en aras a la consecución de los objetivos ambientales fijados en el precepto aludido. Así, este artículo establece diferentes objetivos según se trate de aguas superficiales, subterráneas o zonas protegidas. Para ello fue necesario introducir el artículo 25.a) y b) de la WHG para el caso de las aguas superficiales y aguas artificiales o muy modificadas; a su vez el artículo 25.d) WHG se refiere a las excepciones en la consecución de los objetivos ambientales, tal y como dispone el artículo 4, apartados 5 a 8) DMA; el artículo 32.c) WHG en el que se fijan los objetivos ambientales a alcanzar por las aguas costeras, remite a los dispuesto en los artículos 25.a)-25.d) WHG y, finalmente, en cuanto a la transposición del artículo 4 DMA, el artículo 33.a) WHG establece los fines de los planes hidrológicos de cuenca para las aguas subterráneas.

Los Programas de medidas a los que se refiere el artículo 11 DMA han sido traspuesto en el Derecho alemán a través del artículo 36 WHG, en el que se incluyen todo tipo de medidas de protección de las aguas, así como la prohibición de vertidos directos de contaminantes en las aguas subterráneas,

\footnotetext{
${ }^{56}$ Sobre la discutida naturaleza jurídica de los programas de medidas, vid. CzYCHOwSKi/ REINHARD. Wasserhauhaltsgesetz... Op. cit. Págs. 1290 y ss.
} 
o medidas para la disminución o establecer límites para la introducción de contaminantes por medio de fuentes difusas. La DMA no establece una forma jurídica determinada que estos Programas deban tener, aunque sí el contenido mínimo en algunos casos, como es el artículo 4 DMA o el artículo 11.3) DMA en relación con los requisitos mínimos de las «medidas básicas» que se recogen en los artículos 1 y $2 \mathrm{WHG}^{57}$. Los planes de gestión de las demarcaciones hidrográficas del artículo 13 DMA se traspone a través del nuevo artículo 36.b) $\mathrm{WHG}^{58}$.

La exigencia de una mayor cooperación va a necesitar nuevos mecanismos de transmisión de información no sólo hacia la Unión Europea, sino también entre las diversas autoridades nacionales como confirma LEYMANN ${ }^{59}$. Por ello los necesarios mecanismo de intercambio de información han sido previstos en el nuevo artículo 37.a) WHG.

\section{c) Inciso sobre la transposición de la DMA en los Länder}

Hasta ahora se ha comentado la transposición que en el plano nacional se ha realizado de la Directiva 2000/60, pero aunque no sea éste el objetivo, es necesario hacer un breve inciso en cuanto a los Länder y a cómo esta norma marco europea va afectar a sus Landeswassergesetze ${ }^{60}$, ya que una vez se realizó la trasposición en el nivel estatal en 2002, el siguiente de los pasos a realizar es la trasposición en el plano regional ${ }^{61}$.

Esta adaptación de los Länder al Derecho europeo supone una trascendencia tal que BREUER habla de la Directiva Marco como un caballo de Troya en el sistema federal de la República Federal Alemana ${ }^{62}$ y justamente por ello supondrá para los Länder una disminución de su poder de actuación. Esto se plantea, por ejemplo, en la aplicación del principio de recuperación de costes del artículo 9.1) DMA, puesto que cuando se señala que «Los Estados miembros tendrán en cuenta el principio de la recuperación de los costes de los servicios relacionados con el agua...» no se está teniendo en cuenta la

\footnotetext{
${ }^{57}$ Sobre la situación a fecha de agosto de 2005 de la trasposición de la DMA puede verse la publicación del Bundesministerium für Umwelt, Naturschtuz und Reaktorsicherheit, titulado «Die Wasserrichtilinie- Ergebnisse der Bestandsaufnahme 2004 in Deutschland» en www.bmu.de/gewasserschutz/downloads/doc/35242.php.

${ }^{58}$ Más ampliamente KLuge, T. «Ansätzer zur soziale-ökologischen...» cit. Págs. 18 a 26.

${ }^{59}$ Vid. «Die Bedeutung der Wasserrahmenrichtilinie für den Gewässerschtutz in der Bundesrepublik Deutschland» en Österreichische Wasser- und Abfallwirtschaft. 2001. Pág. 146.

${ }^{60}$ Más ampliamente, KNOPP «Die Umsetzung der EG-Wasserrichtilinie aus der Sicht der Länder» en Zeischrift für Wasserrecht. 4/2003.Págs.1 y ss.

${ }^{61}$ Breuer, R. «Pflicht und Kür bei der Umsetzung der Wasserrahmenrichtlinie» en ZfW. Heft 1/ 2005.Pág. 1.

62 Vid. «Gewässerschutz unf Föderalismus aus der Sich der Wissenchaft» Ponencia en la Jornada «Umweltförderalismus» celebrada en Berlín el 2 de abril de 2001 y organizadas por M. Klöpfer.
} 
vigencia del principio de subsidiariedad, reconocido por otra parte por la Unión Europea en el artículo $5 \mathrm{TCE}^{63}$. Otro ejemplo de la necesaria intervención de los Länder es el artículo 25.c) WHG en el que se dispone que será cada Estado federado, dentro del plazo que la DMA fija - de 22 de diciembre de 2005- el encargado de establecer el plazo para conseguir un buen estado ecológico y químico de las aguas superficiales (de acuerdo con los objetivos del art.25.a) WHG), así como de las masas superficiales artificiales o muy modificadas (según lo dispuesto en el art.25.b) WHG). A estos preceptos anteriores, y también en el caso del art.32.c) y 33.a) WHG deben acompañarse los requisitos que establece el Anexo II y que los Estados federados deben también transponer. Por otra parte el artículo 36.b) WHG dentro de los instrumentos de planificación incluye en el ámbito de Derecho regional (Landesrecht) la determinación del plan hidrológico adecuado a cada cuenca hidrográfica, según los criterios que enumeran en ese mismo precepto ${ }^{64}$.

Los primeros en realizar la reforma de sus Leyes de agua fueron Bayern ${ }^{65}$ y Schleswig-Holstein ${ }^{66}$, así como Rheinland-Pflaz ${ }^{67}$, a los que les siguieron a comienzos del año 2004 Baden-Württemberg ${ }^{68}$ y Bremen ${ }^{69}$, Niedersachsen ${ }^{70}$ , Thüringen ${ }^{71}$, Branderburg en el caso de Hessen el proyecto para trasponer la DMA fue aprobado el 17 de mayo de 2005. De modo que actualmente todos los estados federados ${ }^{72}$ han traspuesto en su ordenamiento al menos el Anexo II de la DMA sobre las características de los tipos de masas de las aguas, y del anexo $\mathrm{V}$ sobre el estado de las aguas, y en ocasiones también el anexo $\mathrm{V}$.

\footnotetext{
${ }^{63}$ Vid. HeINTZEN «Subsidiariätatsprinzip und Europäsiche Gemeinschaft» en JZ. Núm. 7. 1991; ConsTANTINESCO, V. «Le principe de subsidiarité: un passage obligé vers l'Union Européene?» en Europe et le Droit. Mélanges en hommage à Jean Boulois. Dalloz. Paris. 1991. Págs. 39 y ss; entre nosotros, vid. BANDrÉs SÁNCHEZ-CRUZAT, J.M. El principio de subsidiariedad y la Administración Local. Marcial Pons. Barcelona. 1999.

${ }^{64}$ Vid. Sparwasser/ Engel/ Voßkuhle. Umweltrecht. Op. cit. Págs. 518 y 519.

${ }^{65}$ Ley de 24 de julio de 2003. GVB1. Pág. 482.

${ }^{66}$ Ley de 11 de agosto de 2003. GVB1. Pág. 384.

${ }^{67}$ Ley de 16 de octubre de 2003. GVB1. Pág. 309.

${ }^{68}$ Ley de 12 de enero de 2004. GVBl. Pág. 1.

${ }^{69}$ Ley de 18 de diciembre de 2003. GB1. Pág. 401

${ }^{70}$ Ley de 19 de febrero de 2004. GVB1. Pág. 76.

${ }^{71}$ Ley de 18 de abril de 2004. GVB1. Pág. 522.

${ }^{72}$ Más ampliamente sobre la situación real en cada Land, disponible en: www.wrrl-info.de
} 